\title{
Relationship between Oral Health Status and Hematological Values in Pediatric Leukemic Patients: An Evaluative Survey
}

\author{
${ }^{1}$ Karthik Venkataraghavan, ${ }^{2}$ Udita Majithia, ${ }^{3}$ Prashant Choudhary, ${ }^{4}$ Krishna Trivedi, ${ }^{5}$ Shalin Shah
}

\begin{abstract}
Introduction: Leukemia is a malignancy of the bone marrow and constitutes $30 \%$ of all childhood cancers. The leukemic condition itself and its therapy cause oral signs and symptoms with significant morbidity.
\end{abstract}

Aims and objectives: The aim of this study was to review the oral health status in children with leukemia and relate the gingival and periodontal findings to the changes in their hematological values.

Materials and method: The oral health status in 47 pediatric leukemic patients in the age group of 6 to 14 years was assessed using the dmft/DMFT index, OHI(S) index and modified gingival index (MGI). Their hematological reports on the day of examination were obtained. The patients were divided into three groups based on the status of treatment. The relation between the platelet count and the WBC count with the MGI score was checked.

Results: The highest dmf and DMF scores were seen in patients who were currently under treatment. Though an inverse relation was seen between the platelet count and the MGI score, a statistically significant value was not obtained.

Conclusion: A longitudinal follow-up of patients should be carried out in order to establish a relation between the hematological parameters and the gingival inflammation score.

Keywords: Pediatric leukemia, Dental caries, Oral health.

How to cite this article: Venkataraghavan K, Majithia U, Choudhary P, Trivedi K, Shah S. Relationship between Oral Health Status and Hematological Values in Pediatric Leukemic Patients: An Evaluative Survey. J Contemp Dent Pract 2014; 15(5):614-617.

Source of support: Nil

Conflict of interest: None

\footnotetext{
${ }^{1}$ Professor and Head, ${ }^{2}$ Postgraduate Student, ${ }^{3-5}$ Senior Lecturer 1,2,4,5 Department of Pedodontics and Preventive Dentistry College of Dental Sciences and Research Center, Ahmedabad Gujarat, India

${ }^{3}$ Faculty of Dentistry, SEGi University, Malaysia

Corresponding Author: Karthik Venkataraghavan, Professor and Head, Department of Pedodontics and Preventive Dentistry, College of Dental Sciences and Research Centre Ahmedabad, Gujarat, India, Phone: +91 98452 58974, e-mail: veekart@yahoo.co.in
}

\section{INTRODUCTION}

Leukemia is a malignancy of hematologic origin, occurring from either disorganized proliferation or increased life span of white cells (blasts). ${ }^{1}$ Leukemia can be classified basically as lymphoid or myeloid, according to the cell lineage and as acute or chronic, according to the evolution of the disease. ${ }^{2}$ Leukemia constitutes for approximately $30 \%$ of all childhood cancers and acute lymphoblastic leukemia (ALL) is the most common type of malignancy. Nearly, $75 \%$ of childhood leukemia are of the ALL type 3. In India, childhood leukemia constitutes $2.3 \%$ of the cancer patients. Each year 6,000 children in India develop acute lymphoblastic leukemia. ${ }^{3}$

The main characteristic of leukemia is the disordered proliferation of immature or undifferentiated cells in bone marrow and with evolution of the disease, peripheral blood. Due to the growing accumulation of blasts other hematopoietic cells (erythrocytes, macrophages/monocytes, eosinophils, basophils and lymphocytes) are suppressed. As the disease progresses, the excess blast may spread to the bloodstream and infiltrate organs and tissues. ${ }^{2}$

Also observed are severe reductions in the production of platelets and/or the suppression of the normal red cells, which predisposes patients to experience spontaneous bleeding or hemorrhages. ${ }^{4}$ Another change related with leukemia is the increase in the leukocyte count due to an excess amount of blast cells and simultaneous suppression of normal white cells. Once patients have their defenses down, they are at greater risk of developing infections and inflammatory context display is differentiated. ${ }^{4}$

In this scenario, leukemia has been associated with manifestations in the oral cavity such as gingival swelling, petechiae, ulcerations and gingival bleeding. However, studies evaluating oral manifestations in patients with leukemia available in the literature show limitations. Most studies that have explored this area are retrospective analyses or case reports containing information obtained from patient medical records or verified by visual inspections of the oral cavity by healthcare professionals. ${ }^{4}$ Very few studies have explored the association between gingival and hematological parameters with all of them being carried out in the adult population wherein the chronic complications are more frequently seen. ${ }^{2,4,5}$ In the pediatric population where the 
acute complications due to the manifestations of this disease are three times more commonly seen (Alberth et al 2006) ${ }^{6}$ no such study has been carried out. In this sense, consistent evidence to corroborate that the systemic condition of leukemic patients can influence gingival status is currently lacking. On the basis of earlier studies, we hypothesized that the hematological alterations in leukemic patients may induce manifestations in the periodontium. Therefore, the aim of this study was to assess the gingival status of patients with leukemia and identify its correlation with hematological parameters.

\section{MATERIALS AND METHODS}

The present study was carried out in children aged 6 to 14 years who were either newly diagnosed or were under treatment for leukemia at the Pediatric Oncology Ward of Gujarat Cancer Research Institute (GCRI), Ahmedabad. Severely ill, unconscious and uncooperative children were excluded from the study.

After getting the requisite clearance from the GCRI and obtaining written informed consent from the parents, a detailed oral health examination was carried out. Dental health status was recorded with the help of a sterile mouth mirror by a single calibrated examiner. A torch was used as a light source and data was recorded in a modified WHO format. Patients were categorized into three groups based on the status of their treatment:

1. Group I: Patients who were newly diagnosed for leukemia.

2. Group II: Patients who were under chemotherapy/ radiotherapy/combination.

3. Group III: Patients who were under maintenance phase oral cavity was assessed for:

a. Oral hygiene

b. Dental caries

As probing could have induced bleeding, no probes were used. $\mathrm{MGI}^{7}$ was used to record the gingival status. The medical data was obtained from the patients' records. Hematological data were collected from the blood tests performed on the same day as the oral examination. Statistical analysis was done using the Pearson's coefficient of correlation to assess the correlation between the MGI score and the various blood counts, i.e. RBC count, WBC count and platelet count.

\section{RESULTS}

The impact of laboratory parameters on the extent of gingival disease was investigated in order to determine their predictive value and to identify patients who are at an increased risk for such disease.
A total of 47 patients of both sexes were included in the study out of which 3 belonged to group I; 28 belonged to group II and 16 to group III. The mean age of the patients was 10.15 years. Seventy-two percent of the study population were males and 42 out of the 47 patients, i.e. $89 \%$ had ALL. Table 1 shows the means of the dmf, DMF, OHI(S) and MGI scores whereas Table 2 shows the means of the WBC and platelet counts of the three groups divided on the status of treatment (Graphs 1 to 4).

The Pearson's correlation coefficient was used to evaluate the correlation between the following: the mean WBC count and the mean MGI score; the mean platelet count and the mean MGI score. Pearson's correlation coefficient between the means of WBC count and MGI score was $r=$ -0.116 which was not statistically significant $(\mathrm{p}=0.438)$ and that between the means of platelet count and MGI score was $\mathrm{r}=-0.118(\mathrm{p}=0.428)$ which again was not found out to be statistically significant.

\section{DISCUSSION}

Acute lymphoblastic type is a debilitating disease suppressing children's general and dental health, rendering them immunocompromised. Children treated with chemotherapy forming a part of treatment schedule for the same, exhibit acute complications in the oral cavity and long-term complications in dental and craniofacial development. Higher prevalence of dental diseases like dental caries, oral mucositis and gingival inflammation are reported for children with leukemia when compared to systemically healthy children.

In our study the highest $\mathrm{dmf}$ and DMF scores were seen in the group II patients who were currently under treatment $(1.89 \pm 2.30$ and $1.57 \pm 1.73$ respectively) followed by group III patients $(0.00 \pm 0.00$ and $1.57 \pm 1.73$ respectively $)$. This was in accordance to similar studies carried out earlier. ${ }^{3,8}$ This group also showed the highest $\mathrm{OHI}(\mathrm{S})$ score

Table 1: Clinical parameters of the individuals in the study population

\begin{tabular}{llll}
\hline & $\begin{array}{l}\text { Group I } \\
(n=3)\end{array}$ & $\begin{array}{l}\text { Group II } \\
(n=28)\end{array}$ & $\begin{array}{l}\text { Group III } \\
(n=16)\end{array}$ \\
\hline dmf score & $0.67 \pm 0.58$ & $1.89 \pm 2.30$ & $1.06 \pm 1.53$ \\
DMF score & $0.00 \pm 0.00$ & $1.57 \pm 1.73$ & $1.38 \pm 2.13$ \\
OHI(S) score & $0.78 \pm 0.19$ & $1.17 \pm 0.50$ & $1.03 \pm 0.48$ \\
MGI score & $0.95 \pm 0.20$ & $0.29 \pm 0.29$ & $0.33 \pm 0.42$ \\
\hline
\end{tabular}

Table 2: Hematological parameters of the individuals in the study population

\begin{tabular}{llll}
\hline & $\begin{array}{l}\text { Group I } \\
(n=3)\end{array}$ & $\begin{array}{l}\text { Group II } \\
(n=28)\end{array}$ & $\begin{array}{l}\text { Group III } \\
(n=16)\end{array}$ \\
\hline Mean WBC & $11100 \pm$ & $10774.29 \pm$ & $4600 \pm$ \\
count & 2929.62 & 33296.49 & 3434.14 \\
Mean platelet & $32700 \pm$ & $270107.1 \pm$ & $266125 \pm$ \\
count & 6902.656 & 167391.3 & 139807.9 \\
\hline
\end{tabular}




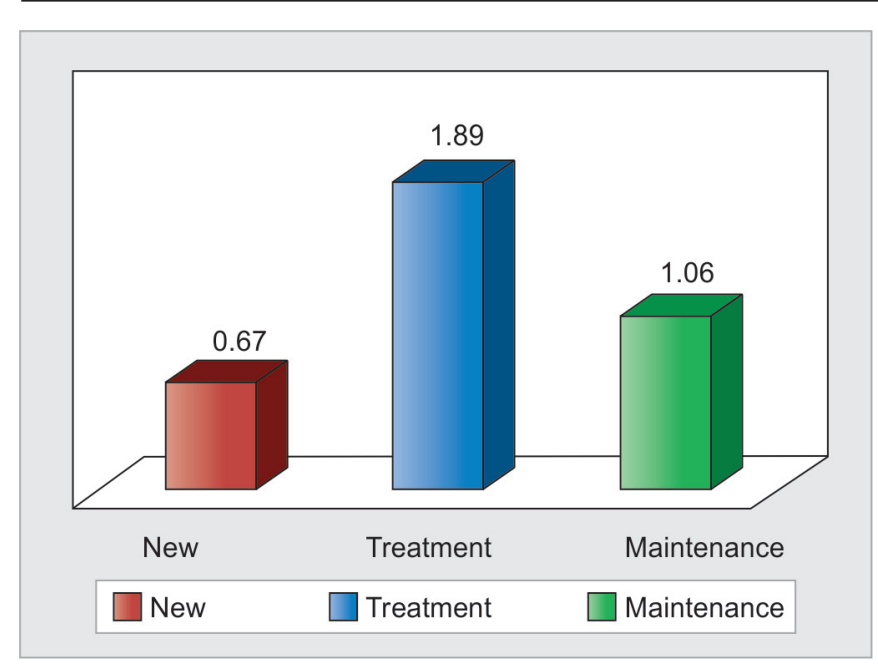

Graph 1: Mean DMF scores of the three groups

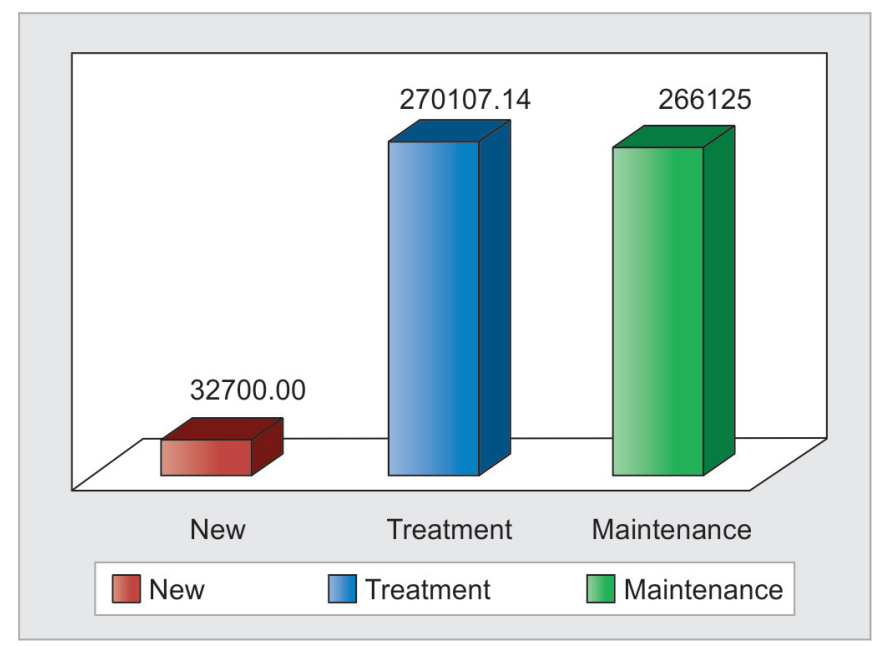

Graph 3: Mean platelet counts of the three groups

of $1.17 \pm 0.50$. This result highlights the fact that the oral hygiene in such patients is usually neglected. This could either be due to the gingival inflammation and bleeding episodes that are associated with the disease or due to the psychology of the patients and the parents and in some cases the treating professionals to treat the systemic condition first. Hence, awareness needs to be spread in this regard that the presence of untreated carious lesions in such patients not only compromises the oral hygiene but also could lead to the spread of infection locally as well as systemically and could also lead to a compromise in the quality of the overall treatment of the systemic disease.

There is evidence that in leukemia, because of the excessive proliferation of blasts in the bone marrow, there is suppression of the normal hematopoietic cells, causing such problems as anemia, thrombocytopenia, leukopenia, infiltration of organs and tissues and others. Specifically regarding thrombocytopenia (low platelet count), an association between increased risk of bleeding and occurrence of hemorrhagic episodes in leukemic patients has been suggested. Though the present investigation did not demonstrate

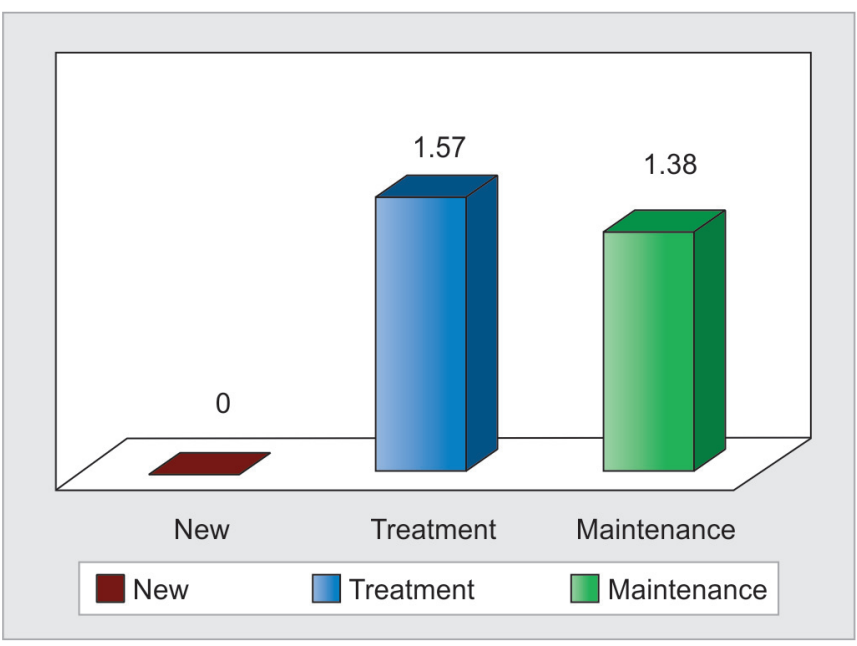

Graph 2: Mean DMF scores of the three groups

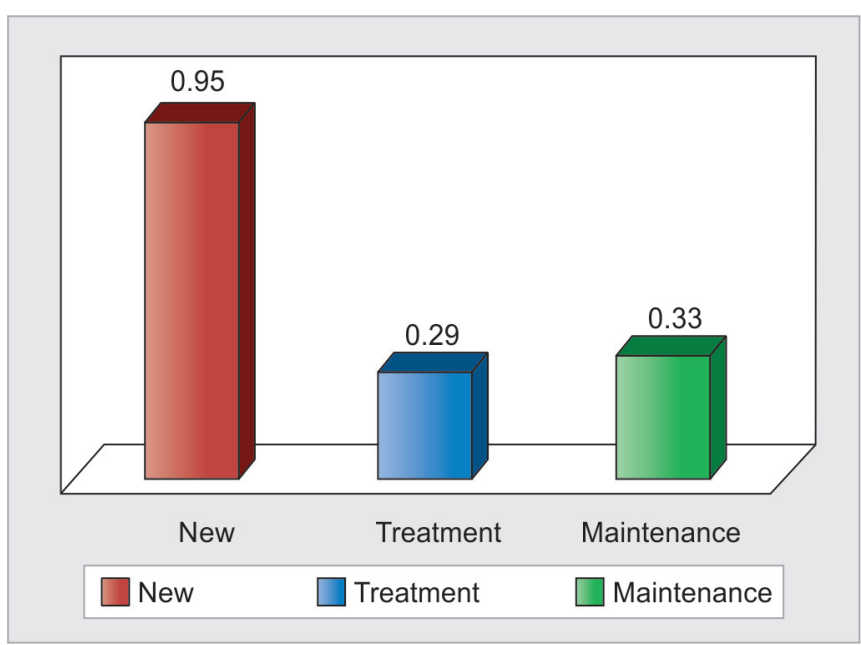

Graph 4: Mean MGI scores of the three groups

a statistically significant correlation between WBC count or the platelet count with the MGI in patients with leukemia we found that the MGI score in group I patients was abnormally high in comparison to their better $\mathrm{OHI}(\mathrm{S})$ scores than the other two groups. This could be due to the extremely low platelet count in this group of patients. Also, we could observe that as the platelet counts improved with treatment there was normalization of MGI scores. The reason for not obtaining a statistically significant relation could be due to the differences in the sample size of the groups and also the variation in the blood counts of the patients. Hence, in order to establish this conclusively a long-term follow-up of more such newly diagnosed cases needs to be carried out.

\section{CONCLUSION}

In the present study, deterioration in the oral hygiene of patients undergoing treatment for leukemia was seen. Hence, awareness needs to be spread about the importance of dental treatment along with the medical management of these patients. Though not statistically significant, a relation was seen between the variation in platelet count and the gingival 
inflammation score, which needs to be established by larger scale longitudinal studies involving more subjects.

Why this paper is important to pediatric dentists?

- Higher prevalence of dental diseases like dental caries, oral mucositis and gingival inflammation are reported for children with leukemia.

- Very few studies have explored the association between periodontal and hematological parameters in children with leukemia.

- Childhood cancer therapy must include oral care, as this will have an impact on the child in the long-term.

\section{REFERENCES}

1. Burke VP, Startzell JM. The leukemias. Oral Maxillofac Surg Clin North Am 2008 Nov;20(4):597-608.

2. Angst PDM, Dutra DAM, Moreira CHC, Kantorski KZ. Gingival inflammation and platelet count in patients with leukemia: preliminary results. Braz Oral Res 2011 Nov-Dec; 25(6):544-549.

3. Nasim VS, Shetty YR, Hegde AM. Dental health status in children with acute lymphoblastic leukemia. J Clin Pediatr Dent 2007 Spring;31(3):212-215.

4. Angst PDM, Dutra DAM, Moreira CHC, Kantorski KZ. Periodontal status and its correlation with haematological parameters in patients with leukaemia. J Clin Periodontol 2012;39(11): 1003-1010.

5. Meyer, et al. Oral findings in three different groups of immunocompromised patients. J Oral Pathol Med 2000;29(4):153-158.

6. Allen G, Logan R, Gue S. Oral Manifestations of cancer treatment in children: a review of the literature. Clin J Onco Nurs 2010 Aug;14(4):481-490.

7. Lobene R, Weatherford T, Ross W, et al. A modified gingival index for use in clinical trials. Clin Prev Dent 1986;8(3):3-6.

8. Hegde AM, Joshi S, Rai K, Shetty S. Evaluation of oral hygiene status, salivary characteristics and dental caries experience in acute lymphoblastic leukemic (ALL) children. J Clin Pediatr Dent 2011 Spring;35(3):319-324. 